\title{
Symbiosis: An Innovative Human-Computer Interaction Environment for Alzheimer's Support
}

\author{
Dimitris Mandiliotis ${ }^{1}$, Kostas Toumpas ${ }^{1}$, Katerina Kyprioti ${ }^{1}$, Kiki Kaza ${ }^{1}$, \\ João Barroso $^{2}$, and Leontios J. Hadjileontiadis ${ }^{1}$ \\ ${ }^{1}$ Dept. of Electrical \& Computer Engineering, Aristotle University of Thessaloniki (AUTH), \\ 54124 Thessaloniki, Greece \\ dimandili@gmail.com, \\ \{ktoumpas, kiaikate, kkaza, leontios\}@auth.gr \\ ${ }^{2}$ Dept. of Electrical Engineering, Universidade de Trás-os-Montes e Alto Douro (UTAD), \\ 5001-801 Vila Real, Portugal \\ jbarroso@utad.pt
}

\begin{abstract}
Alzheimer's disease (AD) is the most common form of dementia diagnosed in people over 65 years of age. There is no cure for the disease, which worsens as it progresses, and eventually leads to death. As the disease advances, symptoms can include confusion, irritability and aggression, mood swings, trouble with language, and long-term memory loss. As the sufferer declines they often withdraw from family and society. Gradually, body functions are lost, ultimately leading to death. Current treatments only help with the symptoms of the disease. Following the vision of WHO and AD International for innovative approaches to $\mathrm{AD}$, the system proposed here, namely Symbiosis, aims at creating a novel human-computer interaction (HCI) environment to facilitate, understand and incorporate the needs of the whole AD community (patients, caregivers and doctors).
\end{abstract}

Keywords: Alzheimer's Disease, Alzheimer's Community, Holistic Approach, Novel Environment, Cloud Database, Augmented Reality, Kinect Games, EEG, GPS Tracking.

\section{$1 \quad$ Introduction}

Human Computer Interaction (HCI) refers to the design and implementation of computer systems that people interact with, including desktop systems, as well as, embedded systems in all kinds of devices [1]. Although the user interface is the primary element between user and computer, HCI is a larger discipline that deals not only with the design of the screens and menus, but with the reasoning for building the functionality into the system in the first place. It is also concerned with the consequences of using the system over time and its effects on the individual, group and company. Stemming from the latter, efficient HCI application in the field of assistive technology for special groups of people (e.g., patients, disabled, elderly) could significantly contribute to the elevation of their quality of life, as it could act as a facilitator for the maximization of the assistance provided by the system to human in a more natural way. The group examined here refers to patients suffering from Alzheimer's disease (AD). 
$\mathrm{AD}$, also known in medical literature as Alzheimer disease, is the most common form of dementia [2]. There is no cure for the disease, which worsens as it progresses, and eventually leads to death [3]. It was first described by German psychiatrist and neuropathologist Alois Alzheimer in 1906 and was named after him. Most often, Alzheimer is diagnosed in people over 65 years of age, although the less prevalent early-onset. Alzheimer develops for an unknown and variable amount of time before becoming fully apparent, and it can progress undiagnosed for years. Current treatments only help with the symptoms of the disease. They cannot stop or reverse the progression of the disease. Alzheimer's disease is also known for placing a great burden on caregivers. Caring for someone with Alzheimer's disease impacts every aspect of daily life. As Alzheimer's patients lose one ability after another, caregivers face pressures wide-ranging from social, psychological and physical elements of the caregiver's life [4]. Moreover, AD is one of the most costly diseases worldwide; the worldwide cost of dementia is estimated to have been US\$ 604 billion in 2010 (Alzheimer's Disease International (ADI) and World Health Organization (WHO) 2012 report) [5], [6]. As the world population ages, we truly face a looming global epidemic of AD. Epidemiological studies indicate that numbers of ADs will nearly double every 20 years, to 65.7 million in 2030 and 115.4 million in 2050, affecting 1 in 85 people globally [7].

Within the aforementioned perspective, Alzheimer's is a global problem with dramatic impact and new approaches need to be considered regarding prevention, diagnosis, treatment, confrontation. Following the guidelines and suggestions of WHO and $\mathrm{ADI}$ for innovative approaches to $\mathrm{AD}$, a new system is proposed here, namely Symbiosis ${ }^{1}$, which aims at creating a novel HCI environment that enhances assistance, facilitates, understands and incorporates the needs of the whole AD community (i.e., patients, caregivers and doctors).

Until now, all technological solutions addressing Alzheimer's focus on the satisfaction of a specific need, e.g., position tracking, memory and skill enhancement and daily needs reminder [8-10]. As the disease progresses, the patient cannot successfully interact with his environment, as the terms that define it becomes obscure. Symbiosis is a revolutionary system aiming at providing integrated solutions to a series of problems related with Alzheimer. Using cutting-edge technology, it provides a holistic approach to the problem, combining all the distinct solutions and introducing a novel HCI strategy that provides a Natural User Interface (NUI) and offers both physical and mental exercise. Symbiosis is the first integrated Alzheimer support system that takes into account the patient's response in an adaptive way that fulfills each patient's special needs and provides considerable facilitations to caregivers and doctors, unlocking the potential of an innovative supporting role.

\section{Design Architecture}

$\mathrm{AD}$ is divided into three stages with progressive patterns [11]:

1 Symbiosis (Greek $\Sigma v \mu \beta i \omega \sigma \iota \varsigma)$, means companionship, from symbioun, to live together, from symbios, living together: syn + bios (life). 
- The preclinical stage with no symptoms (45-55 yrs)

- The mild cognitive impairment or MCI with mild mental symptoms (55-70 yrs), and

- The severe fatal stage of the disease ( $>70 \mathrm{yrs})$

Technology can contribute significantly to the problem of Alzheimer's by offering solutions at the MCI stage of the disease. The most significant skills affected at this stage of the disease are: memory, attention, orientation, visual perception and space perception. Based on this fact, and taking into account design considerations, such as customization, extensibility and user-friendly interfacing, Symbiosis has a modular architecture consisting of three modules with diverse functionalities, i.e., the patient's, the caregiver's, and the doctor's module; all interconnected and exchange information through Microsoft Cloud platform, Azure, [12], in a "symbiotic" way. Fig. 1 depicts the structural architecture of Symbiosis (modules per user's type), where the three pillars (patient (P), caregiver (C) and doctor (D)) are distinguishable, yet lie within a symbiotic space.

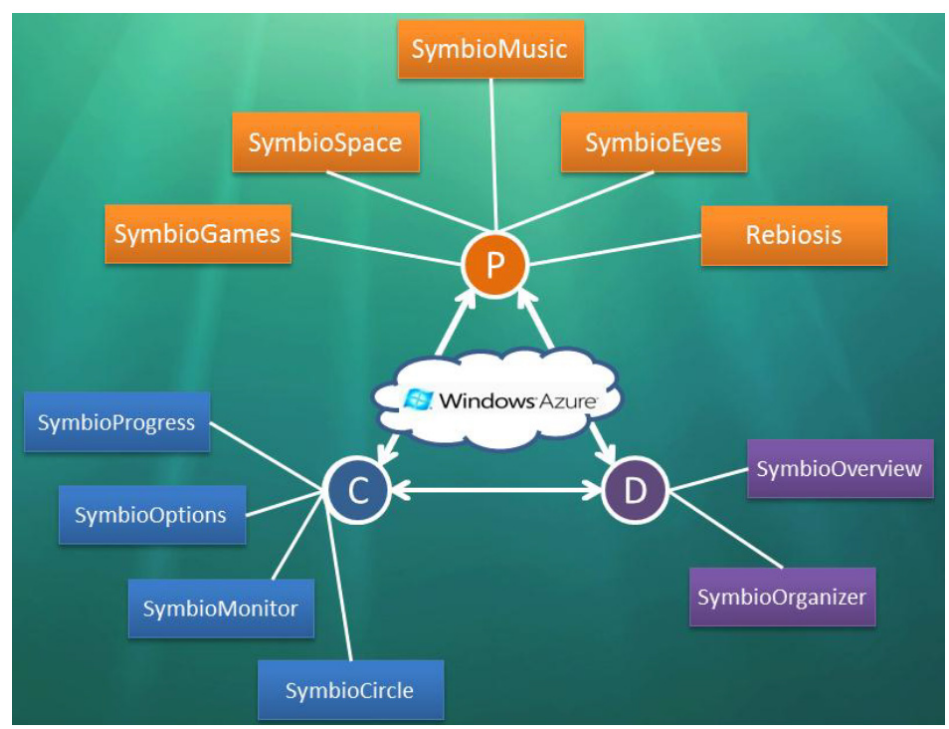

Fig. 1. The modular architecture of Symbiosis and its "Symbiotic" space

\subsection{Symbiosis Patient Module}

The core concept of HCI within Symbiosis is the maximization of the flexibility of user interaction with the system. To this end, Symbiosis proposes a fruitful way of NUI for the patient, through state-of-the-art technologies, such as augmented reality (AR) scenarios, body motion tracking and EEG signal processing. All activities from games to AR scenarios are designed and developed to meet the special needs of an 
AD patient, with special emphasis given on the elimination of social exclusion. Mental and physical exercising, a sense of autonomy and increased self-confidence are the primary focus of all applications along with adaptation to personalized behavior, preferences and needs. In this way, the patient's environment is normalized to his/her needs and not vice versa. In the following subsections, epitomized descriptions of the patient's Symbio-modules (see Fig. 1-the P network) are given.

SymbioGammes. SymbioGames is a gaming suite that offers a wide range of games aiming at exercising and improving skills such as memory, attention, orientation, visual and space perception that gradually decline at the MCI stage of the disease. Furthermore, the novel NUI strategy using Kinect sensor [13] provides a NUI that introduces the idea of combined physical and mental exercise (see Fig. 2).

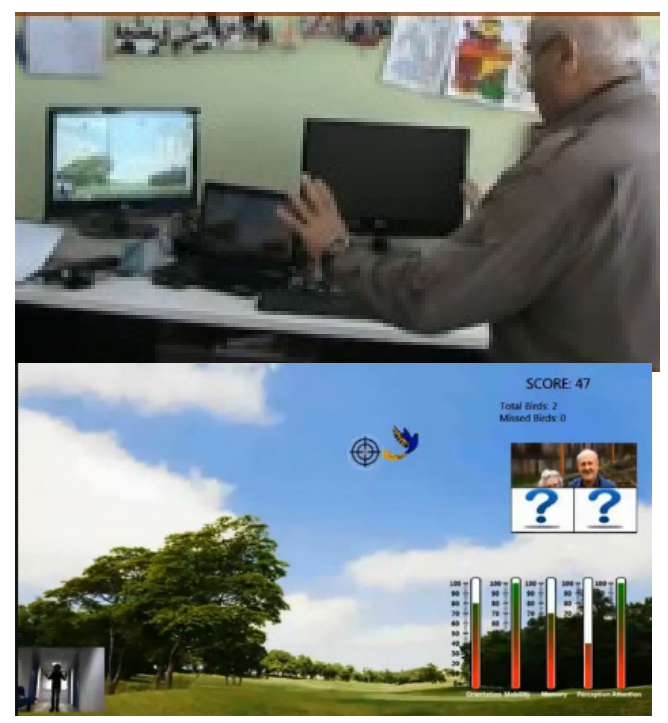

Fig. 2. The Kinect-based NUI [13] of SymbioGames suite (top) when targeting a flying bird and revealing a puzzle-photo from the past (bottom)

SymbioSpace. SymbioSpace application, using auxiliary auditory and visual effects forms a friendly environment for the patient via augmented reality (AR). It offers patient the opportunity to feel surrounded by a helpful environment that provides feedback and seems to interact with him/her responding to his/her needs for continuous reminding and memory refreshing (Fig. 3). 

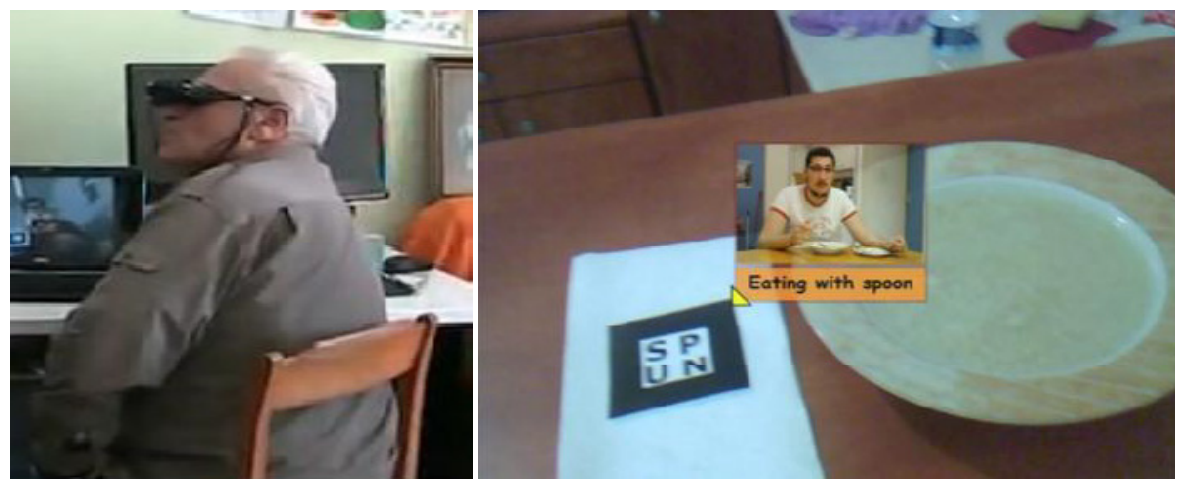

Fig. 3. Using AR glasses (left), SymbioSpace provides to the user additional information for simple tasks, e.g., eating with spoon (right)

SymbioMusic. Music has power-especially for individuals with AD and related dementias. And it can spark compelling outcomes even in the very late stages of the disease. When used appropriately, music can shift mood, manage stress-induced agitation, stimulate positive interactions, facilitate cognitive function, and coordinate motor movements. Based on this fact, SymbioMusic offers a variety of music therapy scenarios that activate musical expression using Kinect sensor combining music revival and memory refreshment.

SymbioEyes. SymbioEyes incorporating automatic photo shooting, GPS tracking, activity reminder, emergency detection and call, is the all-in-one patient mobile application (Fig. 4).

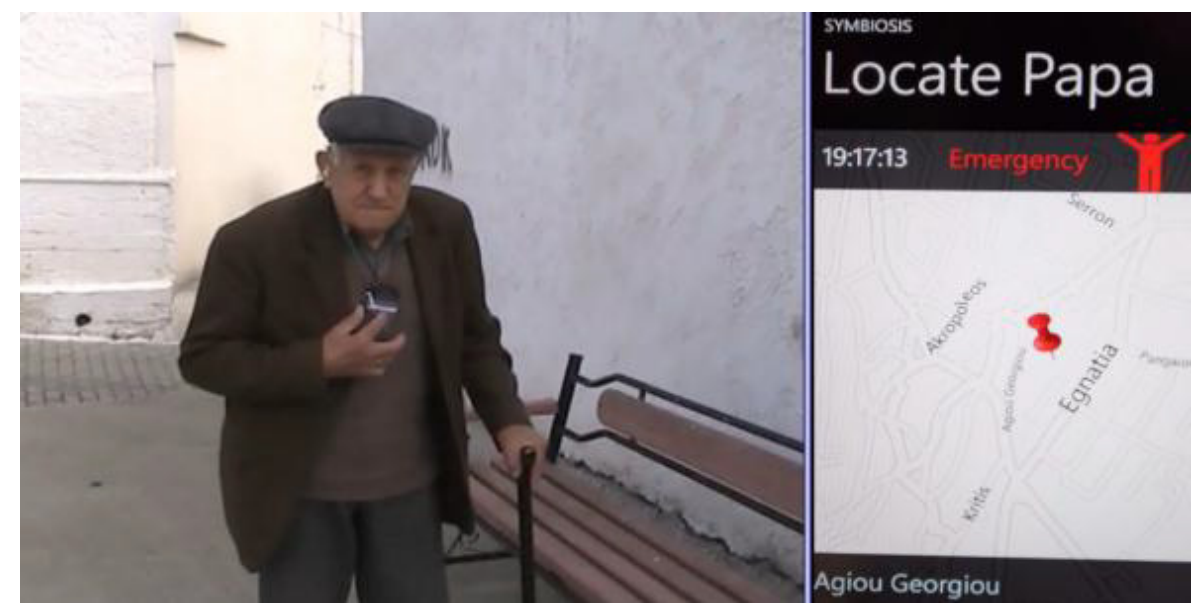

Fig. 4. SymbioEyes monitoring patient's location and status: the user touches the Smartphone surface for more than 2 seconds (left) and the status is changed to Emergency (right) 
Re-Biosis. Re-biosis application downloads automatically from the cloud all photos taken by SymbioEyes application and creates slideshows. While these slideshows are presented to the patient, the photos are subjected to an emotional tagging procedure stemming from EEG signals processing that takes place at the background. The EEG signals are acquired through Emotiv EEG headset [14].

The patient's progress is continuously monitored by both caregiver and doctor, offering valuable feedback information that helps them schedule the therapy plan and daily routine and perform the system appropriate adjustments according to it.

\subsection{Symbiosis Caregiver Module}

Caregiver's every-day life is being supported by the Symbiosis-based local and distant monitoring of the AD patient's behavior, offering emergency notifications and valuable feedback-information that helps schedule his/her daily routine in a novel and efficient fashion. There is emphasis in providing assistance to caregiver for preparation, customization and optimization of patients care and monitoring procedure through a continuous update about their progress via well-managed Web Services. In addition, Symbiosis can be used by the caregiver as a way to come even closer with the broader caregivers' community by sharing and discussing related problems, and so his/her supporting role is transformed to a symbiotic one towards the increase of the AD patients' quality of life. The caregiver's Symbio-modules include:

SymbioSchedule. Balancing the enormous task of care giving with other responsibilities requires diligent planning and organization. SymbioSchedule was developed to meet exactly this requirement. It contains the activity program for the patient as it is scheduled by the doctor.

SymbioProgress. One of the main aspects of care giving is the need for constant monitoring. SymbioProgress constitutes the monitoring tool for the caregiver offering an effective overview of patient's progress, as it is depicted through statistics on patient's interaction with Symbiosis applications (Fig. 5(left)).

SymbioOptions. To achieve personalization and customization of all Symbiosis applications, SymbioOptions provides a control panel where the caregiver can add audiovisual material and adjust the settings of patient's applications.

SymbioCircle. Grief, depression and anger are common among caregivers making psychological support more than necessary. SymbioCircle is Symbiosis forum, where caregivers can find online advice and psychological support as well as exchange opinions with others. 

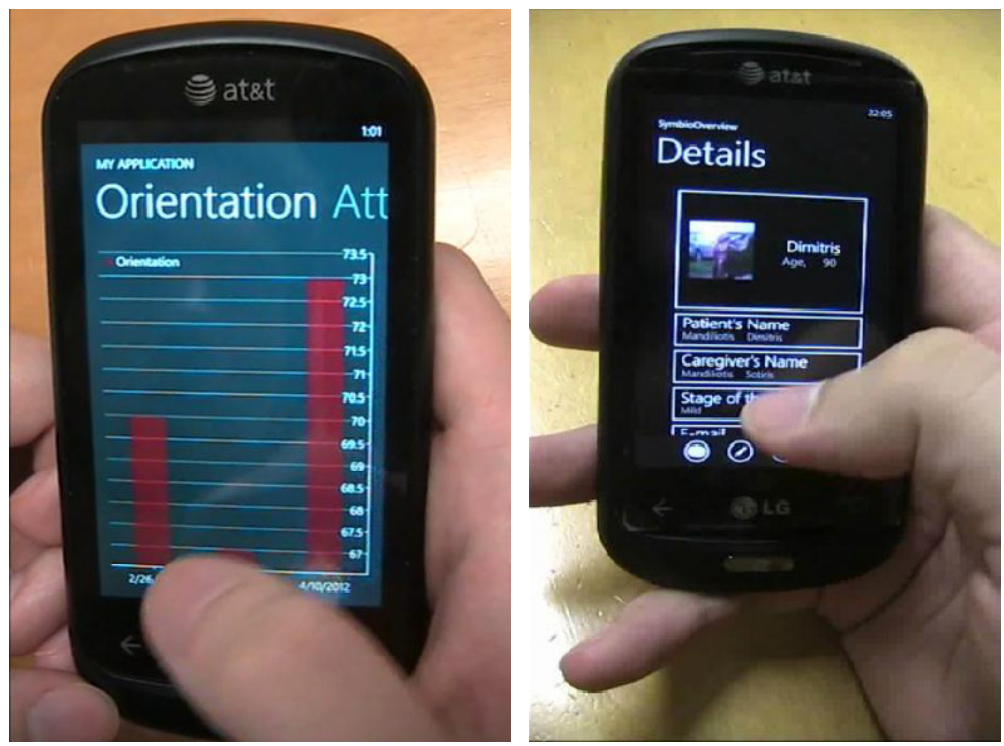

Fig. 5. SymbioProgess (left) provides to the caregiver statistics on patient's interaction with Symbiosis. SymbioOverview (right) provides to the doctor statistics on patient's progress.

\subsection{Symbiosis Doctor Module}

Symbiosis enables the doctor to have daily detailed reporting material with statistical analysis options of every patient's progress, compare the progress of many patients and easily categorize them. Furthermore, the doctor can remotely adjust each Symbiosis' activity parameters according to each patient's profile. Higher levels of functionality and work efficiency are achieved, as Symbiosis takes the computational burden of data analysis, providing all the necessary means for frequent monitoring of the AD patient's health record. Hence, the doctor's working attitude is reformed, as the information of the Symbiosis platform (based on the Microsoft Web-Cloud secure repository) is available anytime and anywhere, setting the handling of the care services on both local (patient's site) and remote bases. The doctor's Symbio-modules include:

SymbioOverview. SymbioOverview enables the doctor to have a detailed and daily statistical overview of every patient's progress, compare the progress of many patients and easily categorize them. Since each doctor has many patients, SymbioOverview enables the doctor to have detailed statistical overview of every patient progress in an easily organized and categorized way (Fig. 5(right)).

SymbioOrganizer. Using SymbioOrganizer, the doctor can adjust the activities parameters according to each patient's profile. 


\section{Experimental Results and Discussion}

A pilot case study of Symbiosis applications involving 20 MCI patients (ages 58-70) was conducted at the two Alzheimer's Care Centers of Thessaloniki, Greece. Applying interaction engagement metrics pre- and post-using Symbiosis, involving: a) successful usages per day/month, b) duration of use, c) recency of use, d) frequency of application abandonments; the results depicted in Fig. 6 have derived. The latter indicate that the use of Symbiosis severely helped the AD patients to increase their spatial perception (mainly due to its NUI) and their interaction engagement (mainly due to the gaming and experiential way of interacting), assisting also to emotional engagement (due to the emotional tagging of images), mental efficiency (due to cognitive games and routing exercises), and memory sustain (due to causality enhancement of actions and relations).

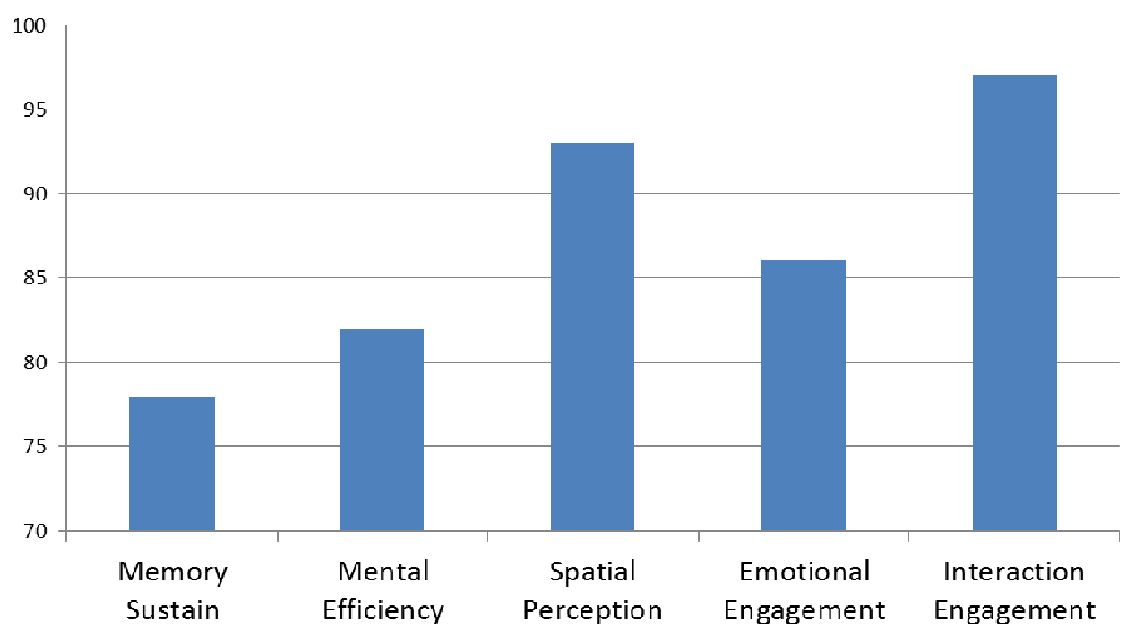

Fig. 6. Mean improvement percentages of five performance indicators between pre- and postusage of Symbiosis by $20 \mathrm{MCI}$ AD patients

Symbiosis is a revolutionary system aiming at providing integrated solutions to a series of problems related with Alzheimer. It is the first integrated Alzheimer support system that takes into account patient's response in an adaptive way that fulfills each patient's special needs and provides to caregivers and doctors considerable facilitations. Until now, all technological solutions addressing Alzheimer's problem, focus on the satisfaction of a specific need. Using cutting-edge technology, Symbiosis provides a holistic approach to the problem which deals with the needs of all groups affected by $\mathrm{AD}$, both literally and metaphorically. Emphasizing on normalization and personalization, Symbiosis proposes a more effective, realistic and complete technological solution. 
Probing to the future, the following issues could be further addressed:

- Cross-case testing and evaluation of the feedback from the target group, regarding the expandability and functionality of Symbiosis, gained through ongoing contact with AD patients, caregivers and doctors at the Alzheimer Center of Thessaloniki and abroad

- Extension of Symbiosis to a multilingual system enabling international use.

- Further increase in the variety of games, exercises and AR scenarios.

- Further customization of tasks and scenarios to different Alzheimer's ages and behaviors.

The Symbiosis concept could also be transferred to other domains, for example, to people with stroke that need rehabilitation (i.e., physiotherapy) scenarios, approached here through a gamification perspective (SymbioGames) to foster users' engagement to the therapeutic task. Moreover, being a facilitator for environment normalization to the user's skills (SymbioSpace), high-level semantics could be extracted from the use of Symbiosis panorama in everyday life of elderly, enabling further research to the identification of possible implicit or hidden correlations between different behavioral expressions or different interpretation styles of the same behavioral perspectives at this age range and study their experiential evolution and transmission from generation to generation or to other communities (for example the behavior change of an $\mathrm{AD}$ patient to his/her children and/or grandchildren).

\section{Conclusion}

Alzheimer's Associations worldwide refer to AD as an epidemic and both medical community and societies agree that Alzheimer's constitutes the great challenge of the 21 century. Symbiosis, by providing innovative solutions that facilitate all the social groups affected by Alzheimer's directly or indirectly, holds the potential to change/improve drastically their lives. Symbiosis provides technological solutions that act as facilitator to the social integration of AD community. The AD patients have greater autonomy; they feel more active through NUI-based gaming/music production and show a collaborative attitude with caregivers, doctors and friends/AD groups. Moreover, caregivers use Symbiosis as an information source about the status of their $\mathrm{AD}$ patients and as a means to come even closer by sharing and discussing related problems with a broader community. Finally, physicians handle more $\mathrm{AD}$ patients and monitor them more closely, increasing their awareness and their assistive intervention in the AD patients' living. Emphasizing on normalization and personalization, Symbiosis proposes a more effective, realistic and complete technological solution.

Acknowledgement. This work has received funding from the EU Seventh Framework Programme FP7-ICT-2011-9-ICT-2011.8.2, under the grant agreement $\mathrm{n}^{\circ}$ 600676: "i-Treasures" Project (http://www.i-treasures.eu). 


\section{References}

1. Card, S.K., Moran, T.P., Newell, A.: The Keystroke-Level Model for User Performance Time with Interactive Systems. Communications of the ACM 23(7), 396-410 (1980)

2. Brookmeyer, R., Gray, S., Kawas, C.: Projections of Alzheimer's Disease in the United States and the Public Health Impact of Delaying Disease Onset. American Journal of Public Health 88(9), 1337-1342 (1998)

3. National Institute on Aging:About Alzheimer's Disease: Symptoms (2011), http: / / www.nia.nih.gov/alzheimers/topics/symptoms

4. Thompson, C.A., Spilsbury, K., Hall, J., Birks, Y., Barnes, C., Adamson, J.: Systematic Review of Information and Support Interventions for Caregivers of People with Dementia. BMC Geriatr., 7-18 (2007), http: / /www.ncbi.nlm.nih.gov/pmc/articles/ PMC1951962/

5. Meek, P.D., McKeithan, K., Schumock, G.T.: Economic Considerations in Alzheimer's Disease. Pharmacotherapy 18(2, pt. 2), 68-73 (1998)

6. Brookmeyer, R., Johnson, E., Ziegler-Graham, K., Arrighi, M.H.: Forecasting the Global Burden of Alzheimer's Disease. Alzheimer's and Dementia 3(3), 186-191 (2007)

7. Förstl, H., Kurz, A.: Clinical Features of Alzheimer's Disease. European Archives of Psychiatry and Clinical Neuroscience 249(6), 288-290 (1999)

8. Lumosity Brain Games, http: / / www. Iumosity. com/sdsd

9. GPS Shoe, http: //www. gpsshoe.com/

10. Pivotell Automatic Pill Dispenser, http: / /www.pivotell.co.uk/index.html

11. Arnáiz, E., Almkvist, O.: Neuropsychological Features of Mild Cognitive Impairment and Preclinical Alzheimer's Disease. Acta Neurol. Scand., Suppl. 179, 34-41 (2003)

12. Microsoft Cloud Platform Windows Azure, http://www.windowsazure.com/ en-us / develop/overview/

13. Gonzalez, A.: Kinect Hardware Review: Wave of the Future. Game Informer (2010), http: / /www.gameinformer.com/b/news/archive/2010/11/03/

kinec-hardware-review.aspx

14. Emotiv EEG, http: //www. emotiv.com 